\title{
Hippotherapy in occupational therapy practice
}

\author{
Pragashnie Govender BOT (UKZN); MOT (UKZN); PhD (UKZN) \\ Lecturer, Occupational Therapy, School of Health Sciences, University of Kwa-Zulu Natal
}

\section{Carol Barlow BOT (UKZN) ** Sameera Ballim BOT (UKZN) **}

** Occupational Therapy Students at the University of KwaZulu-Natal at the time of the study

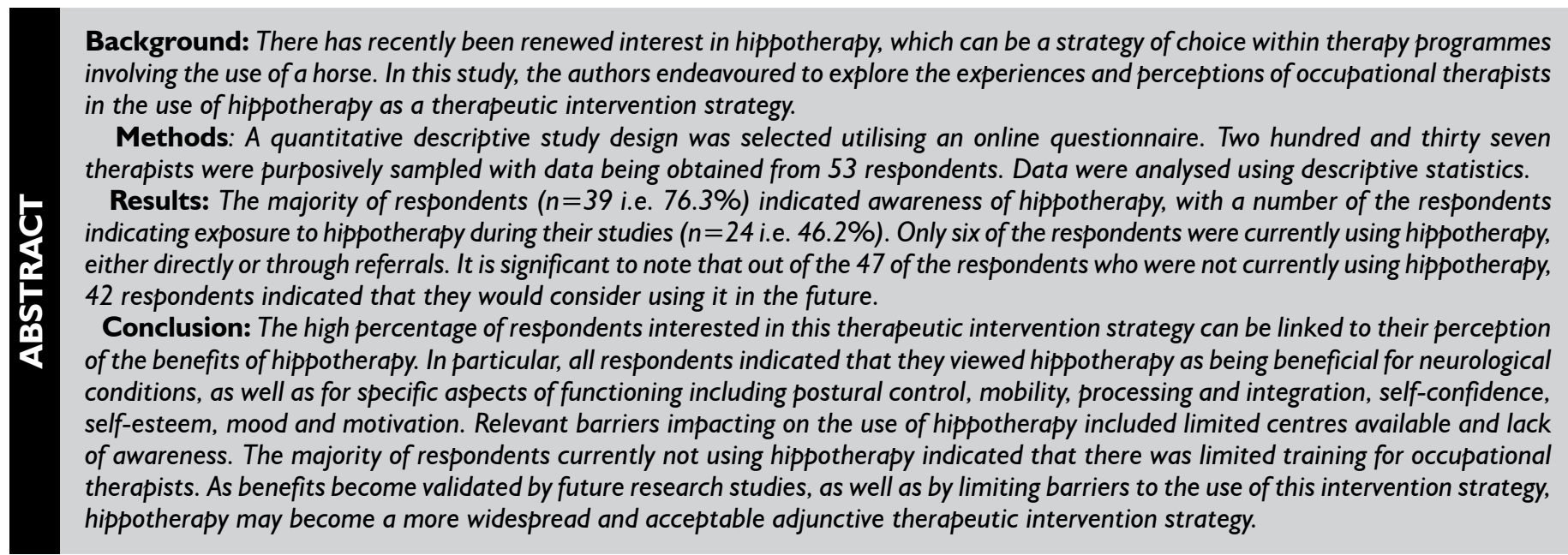

Key words: occupational therapists, occupational therapy, hippotherapy

\section{INTRODUCTION}

"There is something about the outside of a horse that is good for the inside of a man."

Winston Churchill

Hippotherapy is a therapeutic intervention strategy in which the movement of a horse is used as part of an intervention plan. It was initially cited in the in the occupational therapy literature approximately thirty years ago'. There has been renewed interest in this therapeutic intervention strategy recently given the role that occupational therapists play in hippotherapy. Despite this, research evidence that pertains to hippotherapy and its therapeutic outcomes remains limited in the South African context.

It is important to differentiate between the different types of therapies that exist currently with use of the horse. Neill ${ }^{2}$ in her article on horse healing, explains the differences between hippotherapy, developmental riding therapy, psycho-educational vaulting and riding, riding as a sport and skill and equine-facilitated therapy. The focus of this paper is on hippotherapy.

The word "hippos" is Greek for horse, thus "hippotherapy" literally means therapy with the help of a horse ${ }^{3}$. The American Hippotherapy Association ${ }^{4}$ has defined it as "...the use of the movement of the horse as an intervention strategy by physical therapists, occupational therapists, and speech-language pathologists as part of an integrated therapy program to achieve functional outcomes".

In South Africa, hippotherapy is mainly offered on a limited basis by a few skilled therapists or riding instructors under the guidance of a therapist where possible, which contributes to the limited public awareness and involvement.

\section{LITERATURE REVIEW}

Although initially cited in the early 1980's' hippotherapy subsequently received minimal research attention in the field of occupational therapy as the years progressed. Instead studies were carried out by other medical and health professionals largely focussing on the physical effects of hippotherapy. Consequently, recognition grew quickly within the allied health professions and further research studies were undertaken to establish the use of hippotherapy and its physical benefits.

Hippotherapy can be an intervention strategy used by therapists within their therapy programmes to enhance overall occupational performance. Research evidence suggests that therapists may use the horse in a variety of therapy approaches including, amongst others, the neurodevelopmental approach and sensory integration, motor learning, motor control as well as through using psycholinguistic principles. The manner in which the horse is used for individual clients depends on their specific needs, the expertise of the therapist and the training of the horse ${ }^{5}$. Despite this, hippotherapy is regarded as one of the areas with the minimal amount of evidence within the rehabilitation professions ${ }^{6}$.

Hippotherapy has been established in South Africa with two main accredited organisations, namely SARDA (South African Riding for the Disabled Association) (www.sarda.co.za). and SATHRA (South African Therapeutic Riding Association), being pivotal in these developments. Dr Engela Young, an occupational therapist, was the founder of SATHRA ${ }^{7}$ and SARDA was established in 1973 by Belinda Sampson and Joy Finlay. There are currently branches in KwaZulu-Natal (Durban), Port Elizabeth and on the Highveld. 
Benefits of this intervention strategy lie in the horse's unique walk, which provides multidimensional movement, producing approximately "one hundred and ten multi-dimensional movement dialogues in just one minute" ${ }^{8}$. The horse's movements provide rhythmic and repetitive, yet constantly changing sensory input. In order to remain in a stable position on the horse as the horse moves, the client is required to make slight adjustments in his or her trunk. These movement responses reportedly mimic the pelvic movement patterns adopted by humans whilst walking ${ }^{4}$. This duplicated walking pattern sends input to the client's spine and central nervous system and as a result, dormant motor neurons are stimulated and correct movement patterns are recreated. In this way, repetitive application of this intervention strategy is thought to achieve and maintain a more functional brain wave frequency ${ }^{8}$. The variability of the horses gait enables the therapist to grade this degree of sensory input to the client, and then utilise this movement in combination with other therapy strategies to achieve desired results. The client interacts with, and actively responds to the horse's movement. It is documented that the therapist's responsibility is to continuously analyse the client's responses and adjust accordingly thus making the session therapeutic ${ }^{3}$.

A number of studies documenting the physical and psychological benefits of the use of the horse during therapy exists ${ }^{4,9-25}$. Specific benefits reportedly include increasing trunk strength and postural control ${ }^{9,12,19,20,23}$, balance ${ }^{9,12,14,20}$, building overall postural strength and endurance ${ }^{7,11}$, addressing weight bearing, and motor planning ${ }^{4,16}$. It is thus suitably utilised for conditions in which the symptoms present can be addressed through improving strength, balance, overall endurance, and motor planning such as in autism spectrum disorders ${ }^{4,22}$, multiple sclerosis ${ }^{9,11}$, cerebral palsy ${ }^{12,14,15}$, developmental delays $s^{16}$, genetic syndromes ${ }^{4,23}$, learning disabilities ${ }^{15}$, sensory integration disorders $s^{4,16}$ speech-language disorders ${ }^{4}$, spinal cord injuries ${ }^{13}$ and strokes ${ }^{4}$.

In addition to the physical benefits, hippotherapy is believed to have a positive psychological effect ${ }^{17,18,26}$ due to the relationship formed between the client and the horse. This unique relationship allows individuals with emotional disabilities to overcome their fears and develop trust in the horse, the therapist and in their own capabilities. This can lead to increased confidence, patience and self-esteem ${ }^{26}$. Hippotherapy has also been found to foster the motivation that many children with disabilities, having spent extended periods hospitalised or in therapy, no longer possess ${ }^{26}$. The natural environment is also beneficial as it offers challenges associated with being in a non-clinical setting, allowing opportunity for inclusion of community integration intervention ${ }^{4}$.

Despite the above available evidence, hippotherapy is often not clearly understood by individuals who are unfamiliar with the therapy, leading to misunderstanding and misinterpretation. Many regard the word "hippotherapy" to imply a distinctly different and new therapeutic approach or modality, which is as good as, or superior to, other therapies. The fact that the term contains the word "therapy" in it leads to this confusion ${ }^{5}$. It is however, imperative to note that hippotherapy is part of a complete therapy programme. There are various ways the intervention strategy can be utilised ${ }^{5}$. These are inter alia:

* As a preparatory activity, for example, to facilitate increased postural tone in a client who is hypotonic, prior to gait training.

* For mobilisation of spine and pelvis to facilitate assumption of developmental positions during the therapy session to follow.

* As the primary intervention strategy, in order to improve function when off the horse. For example, improvement of sequencing and motor-planning when performing activities on the horse which will contribute to improved performance when off the horse.

Gaps in the Literature: Hippotherapy has been documented as having limited evidence within the rehabilitation professions ${ }^{6}$. Whilst research is growing in the field, particularly in the last two decades, significant gaps remain. Of particular note, is the low level of evidence of existing research studies with reference to study designs, long term effects, and sample sizes. There are also often confounding variables and lack of controls ${ }^{6}$. For example, many clients receiving hippotherapy intervention, also receive other therapy services and it is thus difficult to attribute the benefits identified to hippotherapy alone ${ }^{27}$. Moreover, a significant barrier to hippotherapy research is the difficulty in standardising research conditions, due to the often uncontrollable environment, and standardising outcome measures, particularly for more subjective areas such as emotions and well-being. Further to this, the misinterpretation and blurring of boundaries of "hippotherapy" with "therapeutic riding"6 with the terms being used interchangeably, makes it difficult to identify those which involved therapist-provided treatment.

The authors aimed to explore the status of hippotherapy as a therapeutic intervention strategy within the African context, by identifying the awareness, knowledge and perceptions of occupational therapists in the use of hippotherapy within the province of KwaZulu Natal in South Africa.

\section{METHODS}

Design: A descriptive survey was used. The research population comprised of all qualified occupational therapists within the province of Kwa-Zulu Natal in South Africa, via purposive sampling. The questionnaire was administered electronically to two hundred and thirty seven individuals.

Instrument: A web-based questionnaire using QuestionPro ${ }^{28}$ was used to collect data. This was a self-report questionnaire comprising two sections. Section A (demographic information) involved ten questions. Section $B$, branched into two sub sections. Therapists that were aware of the therapy intervention strategy were automatically directed to Section BI which consisted of a further eleven questions, while those not aware of the intervention strategy completed Section B2 consisting of nine questions. Respondents aware of the intervention strategy but not using it were automatically directed from Section BI to Section B2 in order to answer questions that were appropriate to them. The questions varied between simple yes/no or true/false choices, to multiple tick boxes or Likert scales.

Data Analysis: Microsoft Excel was used to create graphical representations from raw data. Analysis was descriptive which aimed at describing the distribution and range of responses to each variable.

Ethical clearance was granted by the Biomedical Research Ethics committee of the UKZN. In addition, informed consent was obtained from the respondents. The right to withdraw and confidentiality of information was emphasised to respondents.

\section{RESULTS}

This study surveyed respondents that are both users and non-users of hippotherapy as an intervention strategy. The study yielded fifty three respondents, with a low response rate of $22 \%$.

\section{Demographics of Respondents}

All $(n=53)$ respondents were female, ranging between 22 years to 58 years, with the median age being 31 years. Clinical experience of respondents ranged from less than one year to 30 years. More than half of the respondents involved in the study had undergone some form of postgraduate training $(n=35)$ including hand rehabilitation, vocational rehabilitation, research masters, and certifications or training in neurodevelopmental therapy $(n=I I)$, sensory integration $(n=8)$, paediatrics $(n=13)$, neuroscience $(n=2)$, and hippotherapy $(n=2)$. A variety of sectors of practice were represented in this study, including the public sector $(n=24)$, private $(n=14)$, non-governmental organisations or non-profit organisations $(n=3)$, and those that worked in more than one practice setting $(n=6)$. The respondents also represented diverse practice areas, including schools, hospitals, rehabilitation centres, private practices and academic sectors, with varying foci of practice, including physical, psychosocial, vocational rehabilitation, as well as 
community based and assessment-based practice. Clientele age ranges varied, with 23 (42.3\%) of the respondents working with clients across the lifespan, 19 (34.5\%) working specifically with children and adolescents, ten $(21.2 \%)$ respondents working specifically with adults, and one respondent working specifically with clients older than sixty years of age. The respondents also reported working with clients of varying contexts, including rural $(n=10$ i.e $18.9 \%)$, peri-urban $(n=4$ i.e $7.5 \%)$, urban $(n=14$ i.e $26.4 \%)$ and mixed contexts $(n=25$ i.e $47.2 \%)$.

\section{Awareness and Use of Hippotherapy}

The majority of the respondents $(n=39$ i.e. $73.6 \%)$ indicated that they were aware of what hippotherapy involved, whilst 14 (26.4\%) respondents indicated that they were unfamiliar with the term. Also, the majority of the respondents $(n=28)$ indicated that they were not exposed to hippotherapy during their undergraduate studies, whilst the minority $(n=25)$ indicated that they were exposed to hippotherapy during their undergraduate studies. The majority of respondents $(n=47)$ indicated that they were not using hippotherapy in any way (directly or indirectly). Only six $(10.3 \%)$ of the respondents were using hippotherapy. Of these, three of them were using this intervention strategy indirectly (that is, via referrals only and not involved directly) and three respondents were using it directly.

\section{Knowledge of Hippotherapy}

All respondents $(n=53)$ were aware of hippotherapy being a therapeutic medium, utilising the movement of the horse. The majority of respondents was also aware that hippotherapy is not only used for children $(n=50)$, has been proven beneficial for more than just abnormal muscle tone $(n=38)$, is currently used by occupational therapists, speech-language pathologists, physiotherapists and psychologists $(n=46)$ and is conducted by therapists with specific training $(n=45)$. Areas of confusion were noted with 22 (i.e. $41 \%)$ of the respondents believing that during hippotherapy specific horse-riding skills are taught and 29 (i.e 54\%) of the respondents believing that therapists cannot conduct hippotherapy without training if working together with a qualified riding instructor.

\section{Perceptions of Hippotherapy}

This section discusses the perceptions of both users and non-users of hippotherapy in this study. Areas explored include (a) future use of hippotherapy by non-users, (b) benefits of hippotherapy in diagnostic categories according to users and non-users and (c) perceived efficacy of hippotherapy in functional outcomes according to users and non-users.

\section{Consideration of future use of Hippotherapy and mode} of referral (non-users $(n=47)$

Forty two of the 47 respondents not currently using hippotherapy indicated that they would consider using the strategy in the future. The majority of these respondents $(n=26)$ indicated that they would use hippotherapy indirectly by referral, while $I 2$ indicated that they would use it directly working with clients on horses and nine would use it both directly and indirectly (via referrals).

\section{Perceived Benefits of Hippotherapy within Diagnostic Categories}

(i) Benefits reported by current non-users $(n=47)$ of hippotherapy for specific diagnoses

As depicted in Figure $I$, therapists' perceptions of this intervention strategy were as follows. Hippotherapy in the treatment of neurological conditions was well supported with all of this group of respondents
( $n=47)$ agreeing that this intervention strategy would be effective. Psychiatric conditions were also well supported with 42 of these respondents agreeing on the efficacy of hippotherapy in remediation of such conditions. The majority of the respondents also agreed that hippotherapy could effectively treat genetic or congenital conditions $(n=44)$, learning or language disabilities $(n=34)$ and functional spinal curvatures $(n=32)$.

A small percentage of therapists disagreed on the benefits of hippotherapy in some of the aforementioned conditions.

(ii) Benefits reported by current users $(n=6)$ of hippotherapy for specific diagnoses

Respondents currently using hippotherapy were all in agreement on the efficacy of hippotherapy for neurological conditions, learning or language disorders, genetic or congenital conditions and psychiatric conditions (Figure 2). On the contrary, there was disagreement on the efficacy of hippotherapy in addressing functional spinal curvatures with half $(n=3)$ of these respondents believing hippotherapy to be ineffective in treating this condition.

Perceived Benefits of Hippotherapy in Functional Outcomes

(i) Benefits in Functional Outcomes reported by current non-users $(n=47)$ of hippotherapy

Figure 3 indicates the perceived benefits of specific functional outcomes by respondents not currently using hippotherapy. All of the non-users $(n=47)$ believe that hippotherapy could be effective in addressing postural control, mobility, sensory processing and integration, self-confidence and self-esteem, mood and motivation. There was however disagreement regarding its efficacy in addressing strength and endurance, co-ordination, perception, socialisation, attention, concentration and memory, executive functions and behavioural functions. 
(ii) Benefits in Functional Outcomes reported by current users $(n=6)$ of hippotherapy All of the therapists currently using hippotherapy agreed on the efficacy of this strategy in treating postural control, mobility, strength and endurance, sensory processing and integration, co-ordination, perception, self-esteem and self-confidence, socialisation, mood and motivation, attention, concentration and memory, as well as behavioural problems. Two of these therapists however disagreed that hippotherapy is an effective method in addressing executive functions.

\section{Barriers to the use of Hippotherapy}

This section covers the actual and perceived barriers to hippotherapy reported by both users and non-users of hippotherapy.

(a) Barriers reported by non-users $(n=47)$ of Hippotherapy

(i) Actual barriers reported by respondents in the study (non-users)

A large percentage $(89.7 \%)$ of the non-users reported limited knowledge of the strategy to be the main limiting factor. This was followed by logistical inaccessibility ( $n=15$ i.e. $29.5 \%)$, and inaccessibility due to costs $(n=13$ i.e. $26.2 \%)$ as additional barriers. Interestingly, none of the respondents indicated non-use of hippotherapy due to inefficacy of the strategy or because they did not consider it part of the occupational therapy domain.

(ii) Perceived barriers on therapists use of hippotherapy (non-users)

The most common barriers to the use of hippotherapy by occupational therapists, as perceived by those currently using hippotherapy, were logistical difficulties ( $n=35$ i.e. $75 \%$ ), limited training opportunities $(n=35$ i.e. $75 \%)$ available for therapists and limited availability of centres offering the service $(n=35$ i.e. $75 \%)$. About half these respondents $(n=24)$ viewed lack of awareness about the therapy, costs of training in hippotherapy and fear of horses or limited exposure to horses as relevant barriers, whilst 12 respondents viewed cost of implementing this form of therapy as a relevant barrier to occupational therapists.

(iii) Perceived barriers of clients reported by respondents (non-users)

With respect to the barriers for clients, a large number of the nonuser respondents $(n=45$ i.e. $95 \%)$ indicated lack of awareness, limited number of centres available $(n=4 \mid$ i.e. $88 \%)$, logistical difficulties $(n=44$ i.e. $93 \%$ ) and a fear of horses or limited exposure $(n=3 \mid$ i.e.65\%). Cost of the therapy $(n=4 \mid$ i.e. $88 \%)$ was also cited as a major barrier.

(b) Barriers reported by users $(n=6)$ of Hippotherapy All of the respondents who use hippotherapy believed that lack of awareness about hippotherapy, limitations in the number of centres offering this therapy, and logistical difficulties are relevant barriers impacting on the use of hippotherapy by the client. Five of these respondents also believed that fear of horses or limited exposure to horses, as well as the cost of the therapy can also be considered relevant barriers experienced by clients.

The most common barriers to the use of hippotherapy by occupational therapists, as perceived by those currently using hippotherapy, were logistical difficulties $(n=5)$, limited training opportunities $(n=5)$ available for therapists and limited availability of centres offering the service $(n=5)$. Half these respondents $(n=3)$ viewed lack of awareness about the therapy, costs of training in

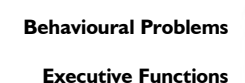

ntration and Memory

Mood and Motivation

Socialisation

Self-Confidence and Self Esteem

Perception

Co-ordination

nsory Processing and Integration Strength and Endurance Mobility

Postural Control
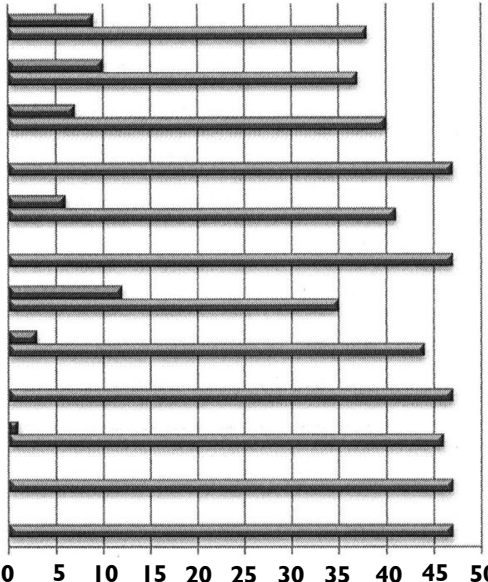

Disagree agree

\section{Number of Respondents}$$
50
$$

utcomes-

non-users $(n=47)$

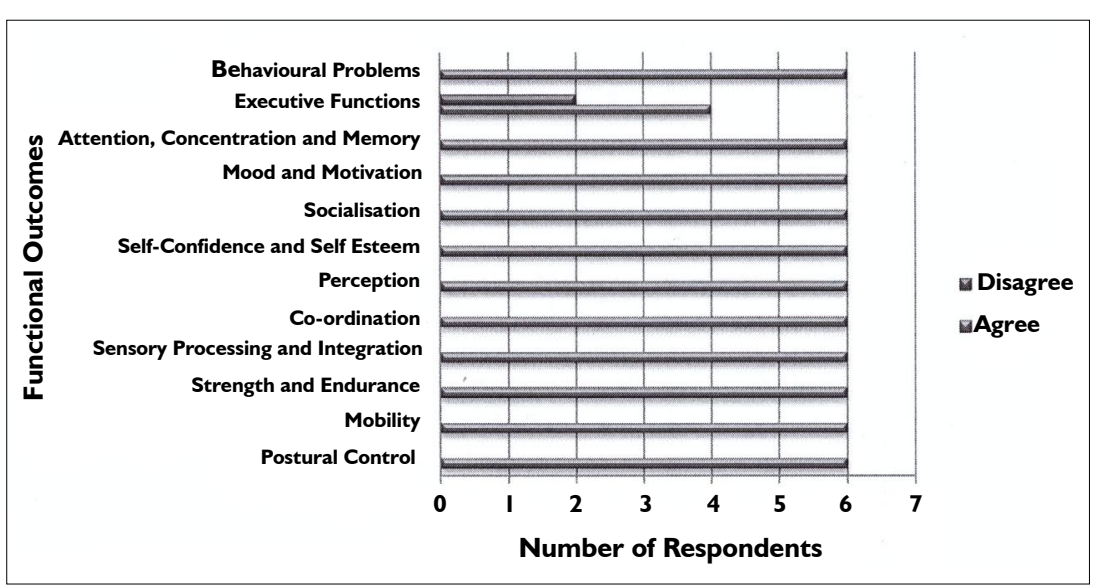

Figure 4: Perceived efficacy of Hippotherapy in functional outcomesnon-users $(n=6)$

hippotherapy and fear of horses or limited exposure to horses as relevant barriers, whilst two respondents viewed cost of implementing this form of therapy as a relevant barrier to occupational therapists.

\section{DISCUSSION}

It is evident that a vast majority of the respondents who were not using hippotherapy indicated that they would consider using hippotherapy in the future. The high percentage of respondents interested in this therapy strategy can be coupled with perceived benefits of hippotherapy. In particular all respondents indicated that they view hippotherapy as being beneficial for neurological conditions, as well as for specific functional outcomes including postural control, mobility, processing and integration, self-confidence and self-esteem and mood and motivation. A significant percentage of the respondents not currently using hippotherapy disagreed on effectiveness of hippotherapy for learning and language disorders and psychiatric conditions. It can be hypothesized that respondents are less convinced about the benefits for these conditions as they are less likely to be associated with the horse's movements. However, research is available to support the efficacy of hippotherapy in these areas. Bazaar (cited in Latella) ${ }^{27}$ found hippotherapy to be an effective tool for speech and language intervention in a 9 year old with autism. Lentini and Knox ${ }^{29}$ conducted a systematic review of studies on the psychosocial benefits of hippotherapy, and results showed decreased feelings of depression and increases in self-esteem, self-confidence and trust. The limited research in these areas is a possible contributing factor to this scepticism. 
Respondents currently using the therapy strategy were more convinced of its benefits in a number of diagnostic categories, however all respondents were not convinced of the efficacy of hippotherapy in addressing functional spinal curvatures. A functional spinal curvature is an abnormal curvature of the vertebral column which is a non-structural or non-fixed abnormality ${ }^{29}$. Borzo's research ${ }^{26}$ however indicated improvements in patients with spinal curvatures with hippotherapy being found to be effective supplementary treatment for patient suffering from scoliosis and more recent research has shown improvements in children with spinal muscular atrophy ${ }^{23}$. Notwithstanding this the research in this area is limited which can be a contributing factor to the scepticism.

In terms of the benefit of hippotherapy on functional outcomes, a number of respondents disagreed with the efficacy of hippotherapy in treating executive functions.

There is however evidence that hippotherapy is able to improve executive functions such as attention, memory, sequencing and following directions through activity on the horse ${ }^{27}$. A drawback when comparing hippotherapy use in normal daily OT practice is the limited accessibility of centres that offer the therapy, particularly within the African context. A large percentage of respondents indicated that the limited training opportunities available to therapists are a significant barrier to the use of hippotherapy, in addition, the limited availability of centres, costs of implementing the therapy including a fear of horses or limited exposure to horses are barriers mentioned. These findings are in keeping with local research findings as in South Africa, there are limitations in the accessibility and viability of hippotherapy as a mainstream therapy ${ }^{8}$. Due to the limited public awareness and limited physical resources, research continues to be limited within an African context with most available studies being conducted internationally. In South Africa, hippotherapy has limited public awareness and involvement ${ }^{8}$. Viability is further affected by the cost of implementing the therapy itself. Overheads of a hippotherapy practice are extremely high, and because riders work on a one-on-one basis, it is difficult to cope with the expenses of establishing a hippotherapy centre ${ }^{8}$. In addition the cost for clients is high particularly when the cost of transport to facilities offering the therapy is taken into account, as these are often out of the central districts. As a result, many cannot afford the fees charged and accessibility is further reduced. However, with increasing information being published on its benefits, this form of therapy is gaining more therapeutic recognition and despite limitations, an increasing number of therapists are becoming involved in hippotherapy ${ }^{8}$.

\section{LIMITATIONS OF THIS STUDY}

An electronic questionnaire was utilised, thus limiting the sample population to persons with available internet access, hence the low response rate. Data within this study is purely descriptive, indicating trends and relationships between variables but not causation. Limited literature is available in this field of study thus providing limited support to findings.

\section{CONCLUSION}

Hippotherapy has evolved over the last thirty years and this intervention strategy has resulted in improved functional outcomes for a variety of clients. This study aimed to describe the awareness, knowledge, perceptions and use of hippotherapy by occupational therapists. Results indicate that a large percentage of persons displayed interest in the use of hippotherapy, yet this therapy intervention strategy is currently only being used by a small percentage of occupational therapists in the province of KwaZulu-Natal. Various barriers have been highlighted as impacting on the use of hippotherapy, the most significant of which include lack of knowledge of hippotherapy, limited training opportunities, logistic barriers, limited centres available and costs of the intervention strategy. As benefits become validated by future research studies, the use of hippotherapy may become a more widespread and acceptable therapeutic intervention strategy. The challenge remains for occupational therapists to provide the evidence of the efficacy of this intervention strategy by engaging in research as well as by accurate and formal documentation. Only then, might hippotherapy be used accurately and therapeutically in the future. With this evidencebased practice, education and clinical experience, therapists may then continue to refine the use of hippotherapy as an adjunct to current therapeutic practices.

\section{REFERENCES}

I. Engel BT. The horse as a modality for occupational therapy. Occupational therapy in health care, I984; I (I): 4I-47.

2. Neill, S. To those who have seen their child healed almost subliminally, the science of therapeutic riding rings true. Horse Healing. Child magazine (undated). Available from: http://www.childmag.co.za/ content/horse-healing\#.V2qTvvn I 7 IV [Accessed 05 June 20I6]

3. Heine $B$ \& Benjamin J. Introduction to Hippotherapy. Advance for Physical Therapists, 2000; II(I3): II-I3.

4. American Hippotherapy Association. [Online] Available from: www. americanhippotherapyassociation.org [Accessed 07 November 2014].

5. Glasow BL. Semantics: To be exuberant or to be correct. American Hippotherapy Association. 2007. [Online] Available from: www.americanhippotherapyassociation.org [Accessed 07 November 2014].

6. Violette K \& Wilmarth MA. Hippotherapy: a therapeutic treatment intervention strategy. Today in PT, 2009: 34-38.

7. Young E, n.d. Dr Engela Young. [Online]. Available from http://www/ engelayoung.co.za/ (Accessed 07 November 2014)

8. Windy Hollow, n.d. Windy Hollow Therapeutic Riding School: Benefits of Horse Movement. Available from: http://www.windyhollow.org.za/Equine_Therapy/Benefits.aspx (Accessed 07 November 2014)

9. Silkwood-Sherer D \& Warmbier H. Effects of hippotherapy on postural stability, in persons with multiple sclerosis: a pilot study. Journal of Neurologic Physical Therapy, 2007; 3 I (2): 77-84.

10. Debuse D, Chandler C \& Gibb C. An exploration of German and British physiotherapists' views on the effects of hippotherapy and their measurement. Physiotherapy Theory and Practice, 2005; 2 I (4): 219-42.

I I. Hammer A, Nilsagård Y, Forsberg A, Pepa H, Skargren E \& Oberg B. Evaluation of hippotherapy (United States). A single-subject experimental design study replicated in eleven patients with multiple sclerosis. Physiotherapy Theory and Practice, 2005; 2I(1): 5I-77.

12. Benda W, McGibbon NH \& Grant KL. Improvements in muscle symmetry in children with cerebral palsy after equine-assisted therapy (hippotherapy). The Journal of Alternative and Complementary Medicine, 2003; 9(6): 817-25.

13. Lechner HE, Feldhaus S, Gudmundsen L, Hegemann D, Michel $D$, Zäch GA \& Knecht H. The short-term effect of hippotherapy on spasticity in patients with spinal cord injury. Spinal Cord, 2003; 4I(9): 502-5.

14. McGibbon NH, Andrade CK, Widener G \& Cintas HL. Effect of an equine-movement therapy program on gait, energy expenditure, and motor function in children with spastic cerebral palsy: a pilot study. Developmental Medicine \& Child Neurology, 1998; 40(II): 754-62.

15. Haehl V, Giuliani C \& Lewis C. Influence of Hippotherapy on the Kinematics and Functional Performance of Two Children with Cerebral Palsy. Pediatric Physical Therapy, 1999; II(2): 99-I0I.

16. Splinter-Watkins KL \& Calhoun SC. Benefits of therapeutic horseback riding: An effective occupational therapy intervention for persons with developmental disabilities. Developmental Disabilities Special Interest Section Quarterly, I999; 22 (4): I-3.

17. Granados AC \& Agis IF. Why children with special needs feel better with hippotherapy sessions: a conceptual review. The Journal of Alternative and Complementary Medicine, 201 I; I7(3): 191-197.

18. Frank A, McCloskey S \& Dole RL. Effect of hippotherapy on perceived self-competence and participation in a child with cerebral palsy. Pediatric Physical Therapy, 20I I; 23(3): 30I-308.

19. Shurtleff TL, \& Engsberg JR. Changes in Trunk and Head Stability after Hippotherapy, a Pilot Study. Physical and Occupational Therapy in Pediatrics, 2010; 30(2): 150-163. 
20. Rigby BR \& Grandjean, PW. The Efficacy of Equine-Assisted Activities and Therapies on Improving Physical Function. The Journal of Alternative and Complementary Medicine, 2015; [Online] Available from: http://online.liebertpub.com/doi/abs/I0.1089/acm.2015.017। (Accessed 05 June 2016).

21. Schneider MS \& Harley LP. The Impact of Therapeutic Riding for People with Disabilities on Variables Related to Mental Health. Anthrozoös, 2016; 29(I):5 9-72.

22. Haggerty $\mathrm{H}$. How Equine Assisted Therapy Can Improve the Quality of Life for Individuals Diagnosed with Autism, Ages 2-18. 2015; Available from: http://digitalcommons.brockport.edu/surc/2015/ schedule/235/ (Accessed 05 June 2016).

23. Lemke D, Rothwell E, Newcomb TM, \& Swoboda KJ. Perceptions of equine-assisted activities and therapies by parents and children with spinal muscular atrophy. Pediatric physical therapy, 20I3; 26(2): 237-244.

24. Kendall E, Maujean A, Pepping CA, Downes M, Lakhani A, Byrne J \& Macfarlane K. A systematic review of the efficacy of equineassisted interventions on psychological outcomes. European Journal of Psychotherapy and Counselling, 20I5; I7(I): 57-79.

25. Rothstein SA. Horses as Healers: The Role of Hippotherapy in Treating Mental and Physical Handicaps. Gleanings; 2013:91.

26. Borzo, G. Horse Power. American Medical News, 2002; 45:24-26.

27. Latella L \& Langford S. Hippotherapy: An Effective approach to OT Intervention. OT Practice, 2008; 13 (2): 16-20.

28. QuestionPro Online Research made Easy. Available from: http:// www.questionpro.com.

29. Lentini JA \& Knox MS. A qualitative and quantitative review of equine facilitated psychotherapy (EFP) with children and adolescents. The Open Complementary Medicine Journal, 2009; I (I):5 I-57.

\section{Corresponding Author}

